\title{
Synthesis of Ordered Mesoporous Silica with Tunable Morphologies and Pore Sizes via a Non-polar Solvent-assisted Stöber method
}

Xiqing Wang ${ }^{\dagger}$, Yu Zhang ${ }^{\dagger}$, Wei Luo ${ }^{\ddagger}$, Ahmed A. Elzatahry ${ }^{\S}$, Xiaowei Cheng ${ }^{\dagger}$, Abdulaziz Alghamdi $^{\#}$, Aboubakr M. Abdullah ${ }^{\xi}$, Yonghui Deng ${ }^{\star \dagger, \psi}$, Dongyuan Zhao ${ }^{\dagger}$

${ }^{\dagger}$ Department of Chemistry, State Key Laboratory of Molecular Engineering of Polymers, and Shanghai Key Laboratory of Molecular Catalysis and Innovative Materials, State Key Laboratory of ASIC \& System, Collaborative Innovation Center of Chemistry for Energy Materials, Fudan University, Shanghai 200433, China

${ }^{\ddagger}$ State Key Laboratory for Modification of Chemical Fibers and Polymer Materials, College of Materials Science and Engineering, Donghua University, Shanghai 201620, China

${ }^{\#}$ Department of Chemistry, College of Science, King Saud University, Riyadh 11451, Saudi Arabia

${ }^{\S}$ Materials Science and Technology Program, College of Arts and Sciences, Qatar University, PO Box 2713, Doha, Qatar

${ }^{\xi}$ Center for Advanced Materials, Qatar University, Doha 2713, Qatar

${ }^{\psi}$ State Key Lab of Transducer Technology, Shanghai Institute of Microsystem and Information Technology, Chinese Academy of Sciences, Shanghai 200050, China. 


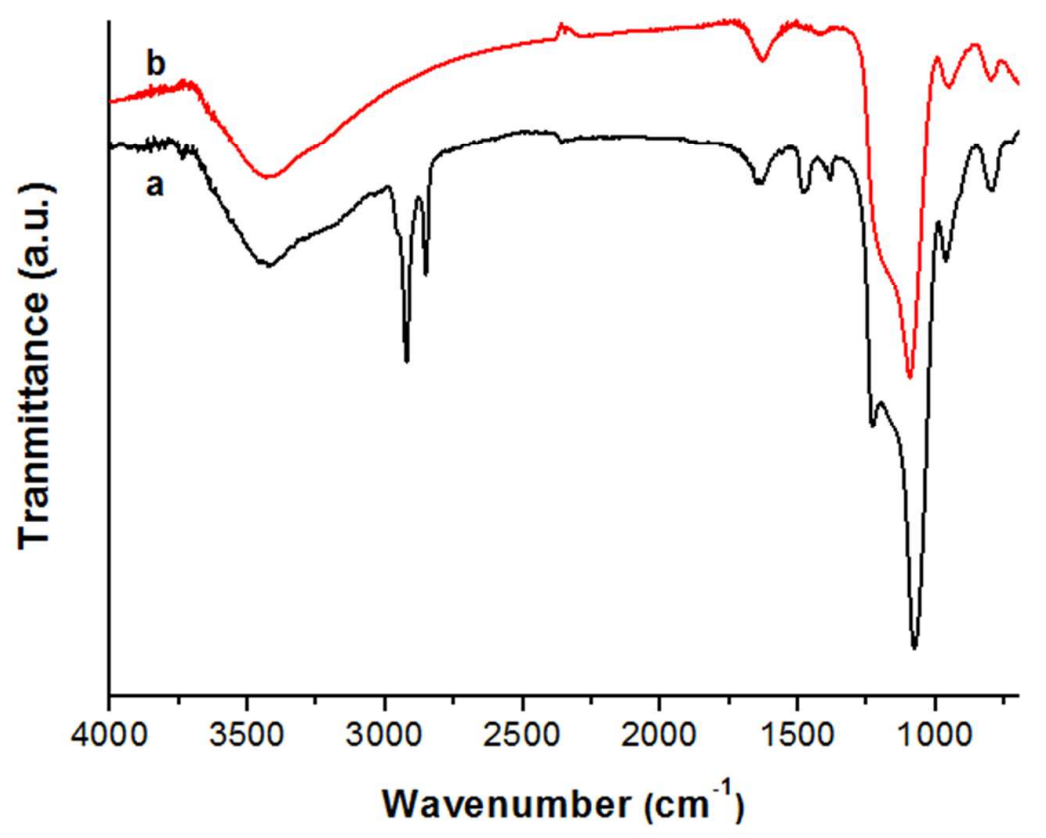

Figure S1. FTIR spectra of (a) the as-made mesostructured $\mathrm{CTAB} / \mathrm{SiO}_{2}$ composite before treatment with ethanol and (b) the obtained mesoporous silica nanocubes obtained after removal of CTAB by ethanol extraction treatment. For the as-made mesostructured $\mathrm{CTAB} / \mathrm{SiO}_{2}$ composite, the bands shown in the region 2800-3000 $\mathrm{cm}^{-1}$ can be attributed to the vibrations of $\mathrm{C}-\mathrm{H}$ bond from CTAB templates. After ethanol extraction, no adsorption peaks were observed in the range of $2800-3000 \mathrm{~cm}^{-1}$ for the obtained mesoporous silica nanocubes, suggesting that the CTAB templates have been completely removed. 


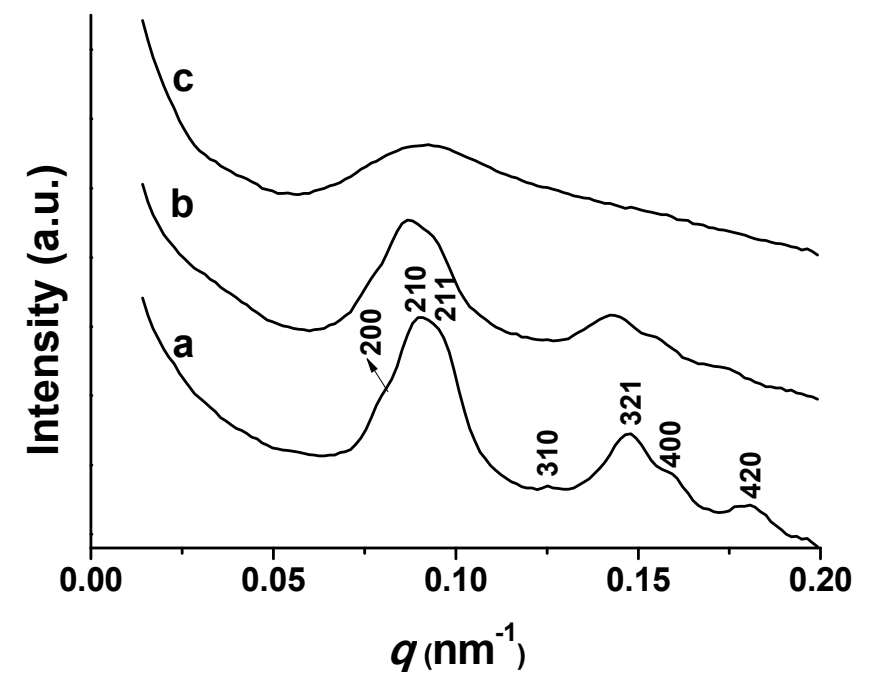

Figure S2. The SAXS patterns of (a) truncated MS-C, (b) MS-S-100 and (c) MS-S-80 synthesized using 5, 3 and $1.5 \mathrm{~mL}$ of concentrated ammonia water, respectively. 

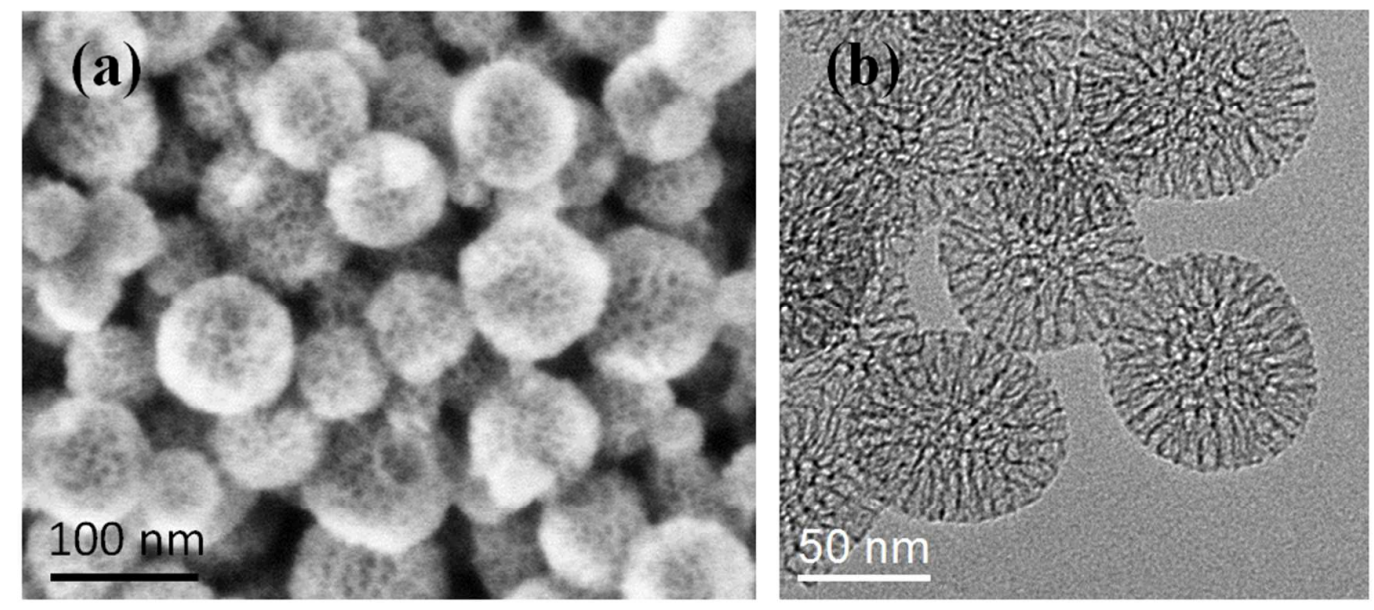

Figure S3. SEM (a) and TEM (b) image of mesoporous silica microspheres (MS-S-80) synthesized using $1.5 \mathrm{~mL}$ of concentrated ammonium water. 

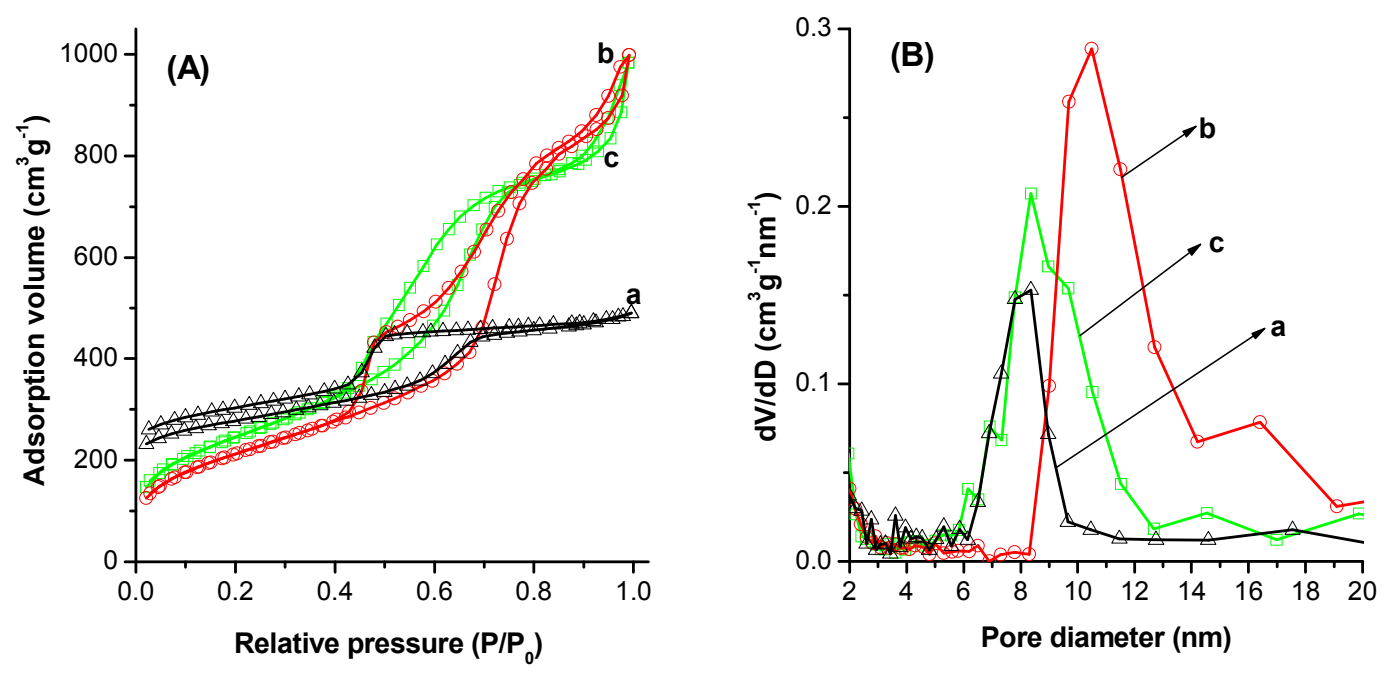

Figure S4. (A) Nitrogen adsorption-desorption isotherms and (B) pore size distributions of (a) truncated MS-C, (b) MS-S-100 and (c) MS-S-80 synthesized using $5 \mathrm{~mL}, 3 \mathrm{~mL}$ and $1.5 \mathrm{~mL}$ of concentrated ammonia water, respectively. The pore size distributions were calculated using Broekhoff de Boer (BdB) spherical model from the adsorption branches.

Table S1. Textural properties of the obtained truncated MS-C, MS-S-100 and MS-S-80 samples

$$
\mathrm{S}_{\mathrm{BET}}\left(\mathrm{m}^{2-1} \mathrm{~g}^{-1} \quad \mathrm{~V}_{\text {total }}\left(\mathrm{cm}^{3} \mathrm{~g}^{-1}\right) \quad \text { Pore size }(\mathrm{nm})\right.
$$

\begin{tabular}{cccc}
\hline truncated MS-C & 620 & 0.90 & 8.3 \\
MS-S-100 & 774 & 1.55 & $10.5(16.3)$ \\
MS-S-80 & 888 & 1.52 & $8.4(14.5)$
\end{tabular}

The values in the bracket indicate the larger pores in the shell of the MS-S samples. 


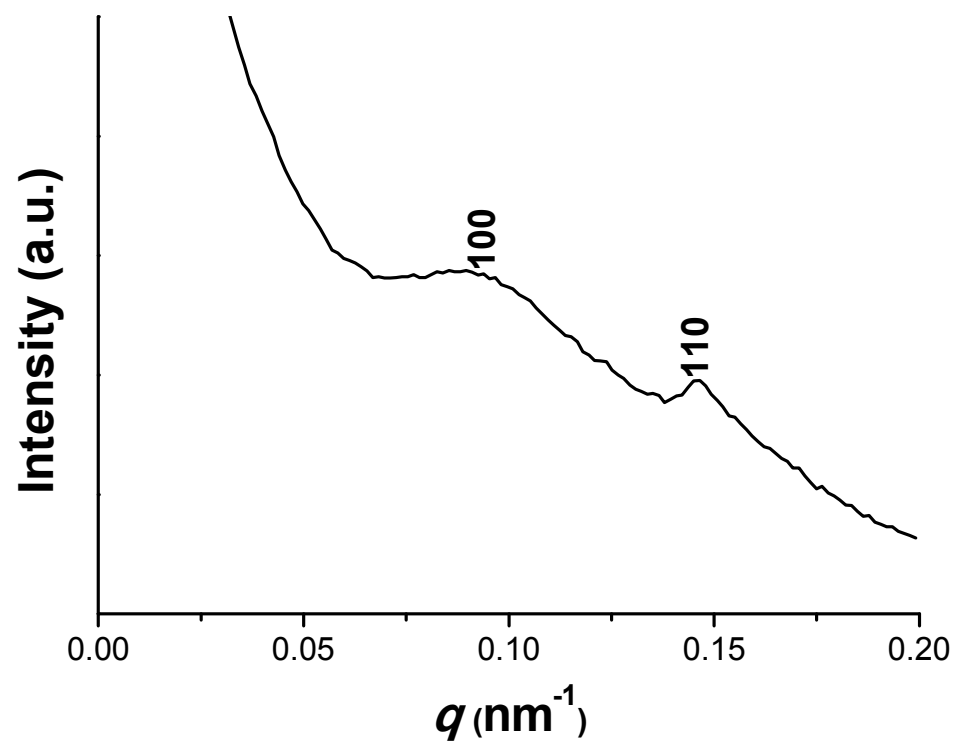

Figure S5. The SAXS pattern of the twisted mesoporous silica rod (MS-R) synthesized using $20 \mathrm{~mL}$ of concentrated ammonia water and $6 \mathrm{~mL}$ of $n$-hexane. 


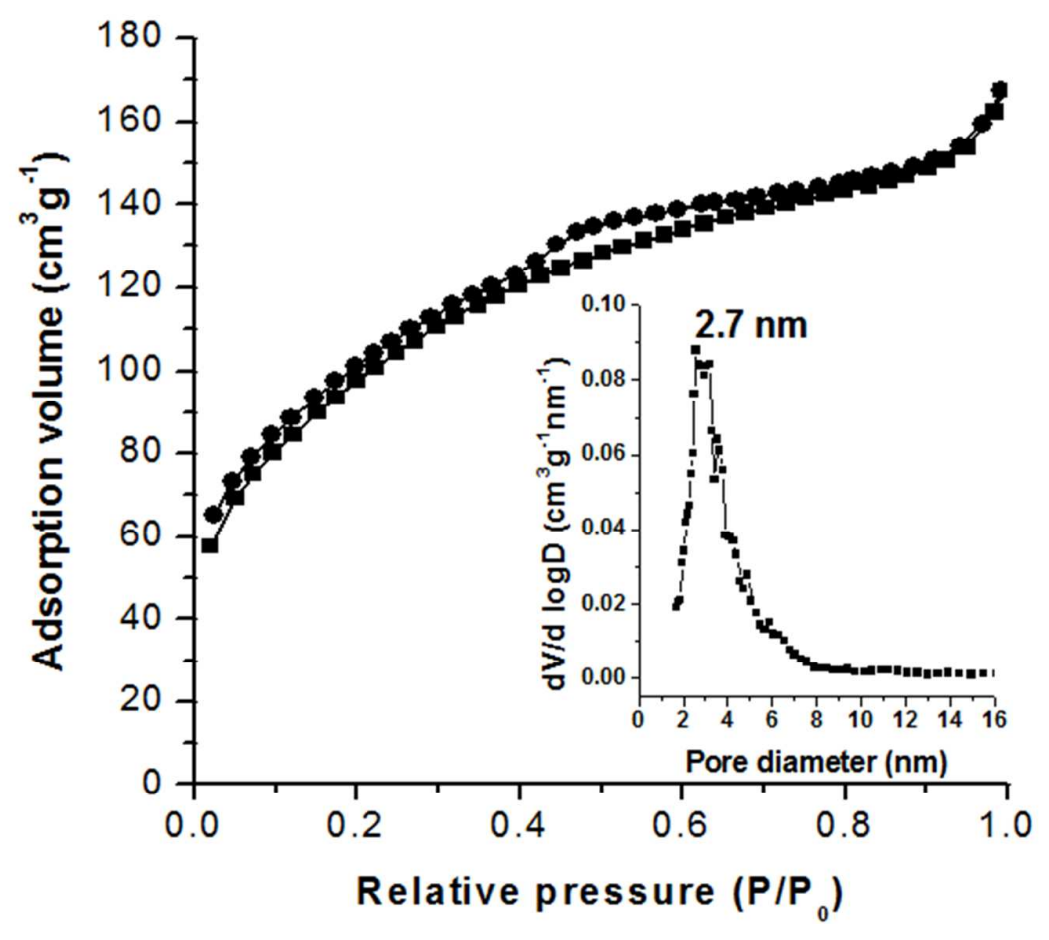

Figure S6. Nitrogen adsorption-desorption isotherms and the pore size distribution (the inset) of the twisted mesoporous silica rod (MS-R) synthesized using $20 \mathrm{~mL}$ of concentrated ammonia water and $6 \mathrm{~mL}$ of $n$-hexane. 


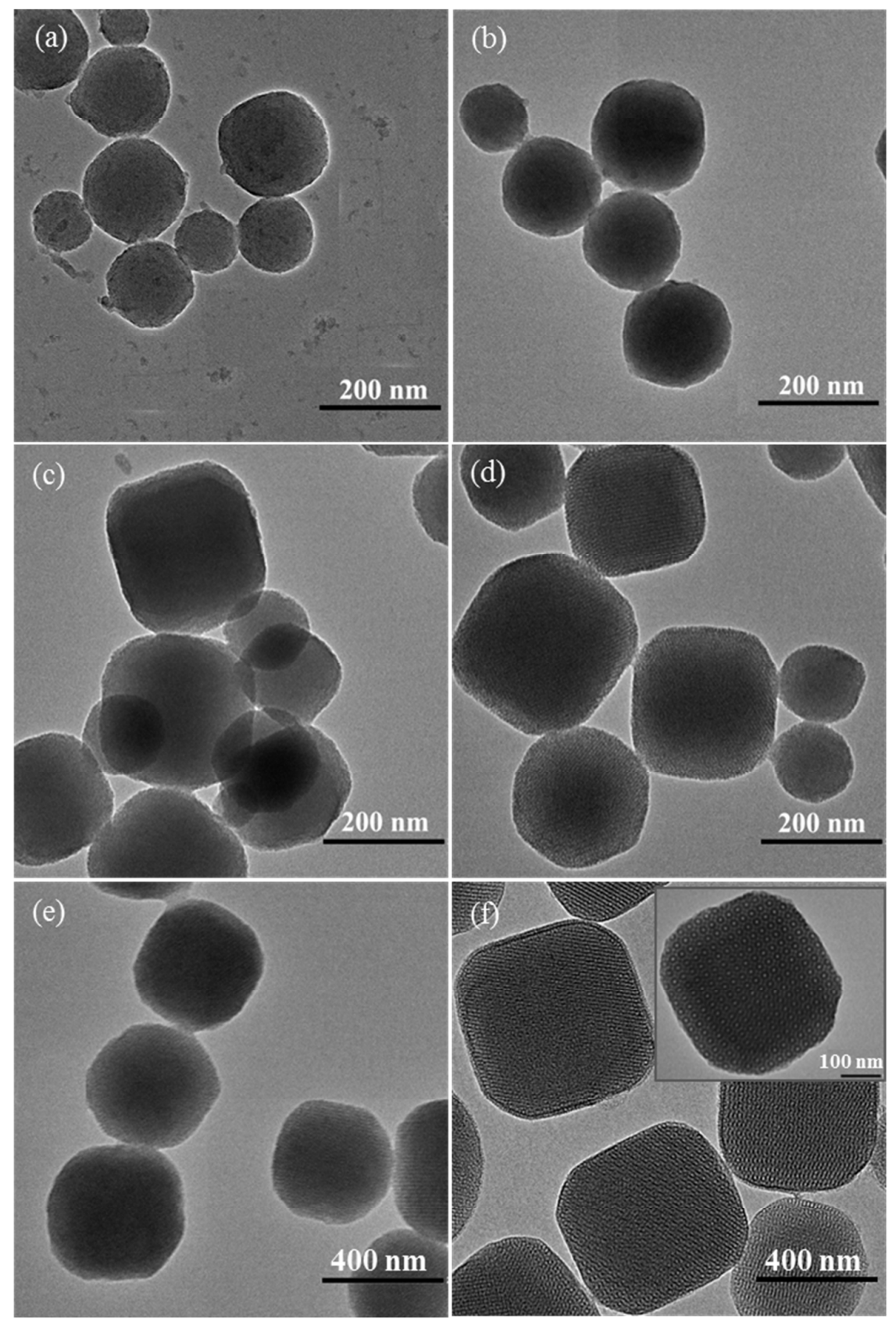

Figure S7.TEM images of samples withdrawn from the synthesis solution during the synthesis of MS-C at different time, (a) $0.5 \mathrm{~h}$, (b) $1 \mathrm{~h}$, (c) $3 \mathrm{~h}$, (d) $5 \mathrm{~h}$, (e) $7 \mathrm{~h}$, (f) $10 \mathrm{~h}$. The inset in (f) was viewed along [100] direction. 


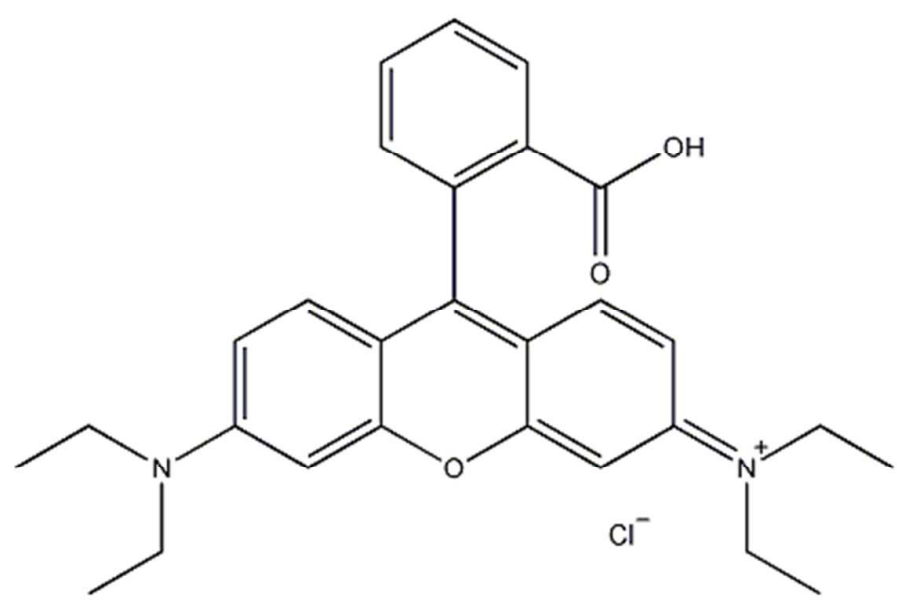

Figure S8. The molecular structure of Rhodamine B with a molecular dimension of $1.59 \times 1.18 \times 0.56 \mathrm{~nm}$. 

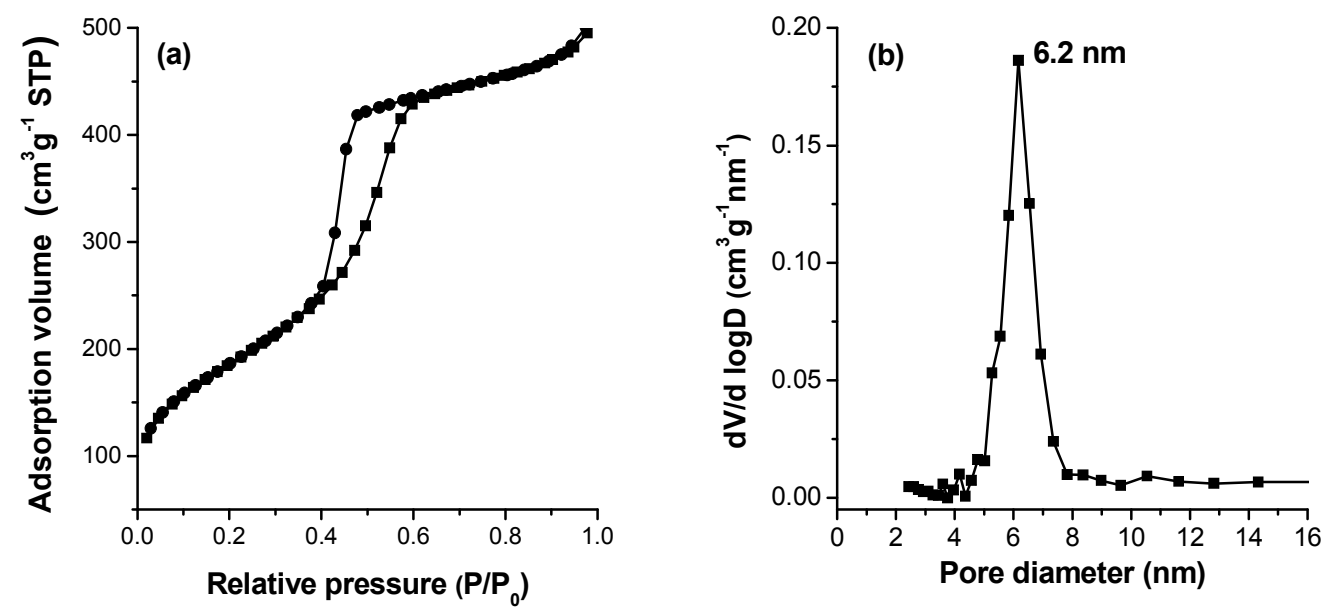

Figure S9. Nitrogen adsorption-desorption isotherms and corresponding pore size distribution of C-MS-C obtained by calcination of the as-made CTAB/silica composite with a cubic mesostructure instead of solvent extraction for removal of CTAB.

Table S2. Textural properties of the obtained MS-C and C-MS-C obtained by solvent extraction and calcination in air, respectively

\begin{tabular}{lccc}
\hline & $\begin{array}{c}\text { Total pore volume } \\
\left(\mathrm{cm}^{3} \mathrm{~g}^{-1}\right)\end{array}$ & $\begin{array}{c}\text { Mesopores size } \\
(\mathrm{nm})\end{array}$ & $\mathrm{S}_{\mathrm{BET}}\left(\mathrm{m}^{2} \mathrm{~g}^{-1}\right)$ \\
\hline MS-C & 1.06 & 7.8 & 801 \\
C-MS-C & 0.79 & 6.2 & 667 \\
\hline
\end{tabular}




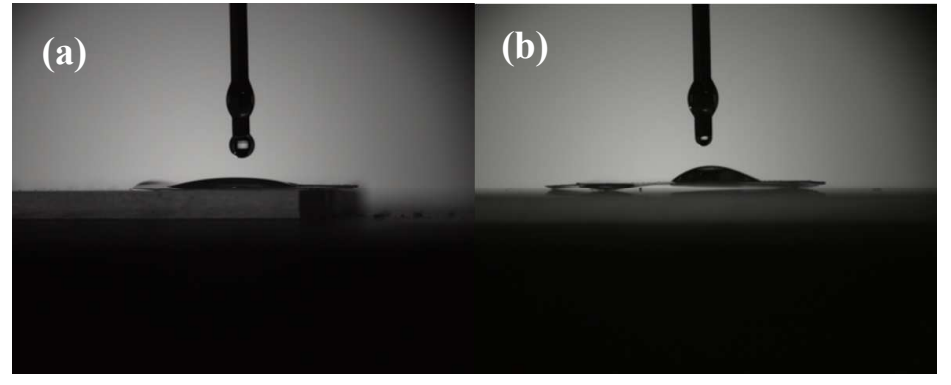

Figure S10. Photographs of water droplet on a MS-C pellet with contact angle of $17^{\circ}$ (a), water droplet on a C-MS-C pellet with contact angle of $39^{\circ}$ (b). 